\title{
Topology Design of Pressure Adaptive Honeycomb for a Morphing Fowler Flap
}

\author{
Jan Scheepstra* and Roelof Vos ${ }^{\dagger}$ \\ Delft University of Technology, Delft, The Netherlands \\ Ron Barrett ${ }^{\ddagger}$ \\ The University of Kansas, Lawrence, Kansas
}

\begin{abstract}
A new method for designing a morphing Fowler flap based on pressure-adaptive honeycomb is detailed. Pressure adaptive honeycomb has been shown to be able to induce gross camber deformations in airfoil sections, such as a flap. However, due to the large amount of design variables the integration of the honeycomb as a distributed actuator in a flap structure has proven to be challenging. Therefore, a design tool is developed that can aid the designer in generating a honeycomb and flap topology that ensures two desired shapes under two distinct conditions: a cruise shape and a high-lift shape. This tool is based on a finite-element analysis of the pressure-adaptive honeycomb, where the honeycomb elements are reduced to simple rigid-bar elements connected by frictionless hinges. This tool is verified against analytical and experimental results. In the present implementation the honeycomb is attached to the upper skin of the flap, which itself introduces curvature in the aft flap section. An optimization algorithm calculates the exact thickness distribution of the skin that introduces the prescribed shape in high-lift conditions. By varying the pressure inside the honeycomb, the initial skin curvature distribution, or the honeycomb topology, the designer can evaluate how well the calculated shapes match the desired shapes in high-lift and cruise condition. This allows for a faster and more accurate topology designs that can ultimately enable an effective morphing solution to increase the maximum lift capability of Fowler flaps.
\end{abstract}

\section{NOMENCLATURE}

A Element cross sectional area, $\mathrm{m}^{2}$

$C_{p} \quad$ Aerodynamic pressure coefficient, -

E Young's modulus, $\mathrm{N} / \mathrm{m}^{2}$

$\bar{f} \quad$ External force vector, -

F $\quad$ Force, $\mathrm{N}$

I Second moment of area, $\mathrm{m}^{4}$

k Element stiffness matrix, -

K Global stiffness matrix, -

$l \quad$ Cell wall length, $\mathrm{m}$

$L \quad$ Element length, m

$M \quad$ Mach number or moment, -, Nm

$N \quad$ number of data points or bar tension, -, N

$p \quad$ Pressure, $\mathrm{N} / \mathrm{m}^{2}$

$\bar{p} \quad$ Internal force vector, -

$q \quad$ Dynamic pressure, $\mathrm{N} / \mathrm{m}^{2}$

${ }^{*}$ Graduate Student, Faculty of Aerospace Engineering at Delft University of Technology, Kluyverweg 1, 2629HS Delft, The Netherlands

${ }^{\dagger}$ Assistant Professor, Faculty of Aerospace Engineering at Delft University of Technology, Kluyverweg 1, 2629HS Delft, The Netherlands, AIAA memeber

${ }^{\ddagger}$ Associate Professor, Department of Aerospace Engineering, 2120 Learned Hall, Lawrence, KS 66045, AIAA member 
$\bar{r} \quad$ Global residual force vector, -

Re Reynolds number, -

$t \quad$ Cell wall thickness, $\mathrm{m}$

$U \quad$ Internal energy potential, -

$\bar{u} \quad$ Nodal displacement vector, -

$V \quad$ Volume or External energy potential, $\mathrm{m}^{3}$, -

\title{
Greek Symbols
}

$\begin{array}{ll}\alpha & \text { Angle of attack, deg } \\ \delta & \text { Deflection angle, deg } \\ \varepsilon & \text { Error, - } \\ \theta & \text { Cell wall angle, rad } \\ \varphi & \text { Skin element angle, rad } \\ \Pi & \text { Total system energy, - }\end{array}$

\begin{tabular}{ll}
\multicolumn{2}{l}{ Subscripts } \\
0 & Initial \\
$\infty$ & free stream conditions \\
av & Average \\
aero & aerodynamic \\
CDP & CDP loading \\
eq & Equivalent \\
$i$ & Initial or index \\
$f$ & flap \\
$g$ & geometric \\
$l$ & load \\
$m$ & material \\
rot & rotational stiffness \\
skin & bending
\end{tabular}

\author{
Abbreviations \\ CDP Central Differential Pressure \\ PAH Pressure Adaptive Honeycomb \\ TL Total Lagrangian
}

\section{INTRODUCTION}

Aircraft wing design is generally driven by two disparate design requirements: a maximized lift coefficient during landing and a maximized lift-to-drag ratio during cruise. To accommodate both requirements, aircraft are traditionally equipped with high-lift devices, in particular flaps and slats. These devices have been shown to increase the lift coefficient of aircraft significantly. Their additional weight, complexity, and cost penalties have historically been outweighed by their performance gain. In an effort to reduce part count, complexity, and cost of conventional high-lift devices a new type of adaptable structure has been conceived. Because none of the conventionally accepted adaptive materials like shape memory alloys or piezoelectric ceramics possess FAA certifiable databases, this novel adaptive structure is based on conventional, certified aerospace materials. This structure relies on a grid of conventional, hexagonal honeycomb cells that extend over a significant length perpendicular to the plane of the cells. Inside each of the cells a pouch resides which can be inflated such as to form a circular tube inside the hexagonal honeycomb cells. By varying the so-called cell differential pressure (CDP) the overall stiffness of the pressurized honeycomb can be varied. With the 
addition of external or internal restoring forces this stiffness variation translates to a structure that can deform as a function of the CDP. The high level of compliance of the honeycomb ensures overall elastic strains exceeding 50\%, an order of magnitude higher than for example SMAs. Figure 1 shows the principle of pressure-adaptive honeycomb for a flap-based test-article.

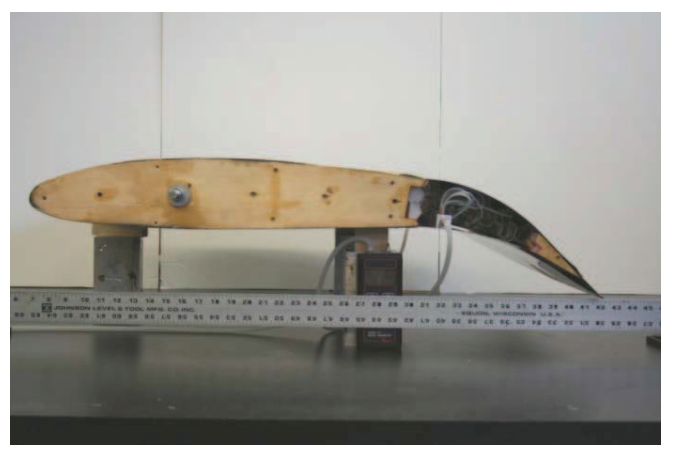

(a) $\mathrm{CDP}=0 \mathrm{kPa}$

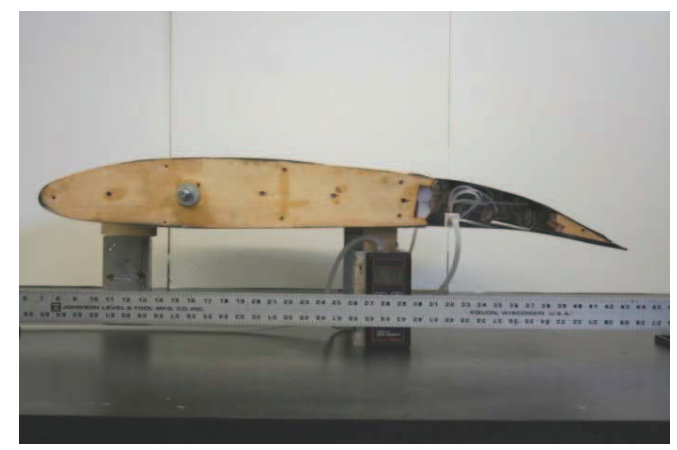

(b) $\mathrm{CDP}=40 \mathrm{kPa}$

Figure 1. Example of Pressure Adaptive Honeycomb in a Morphing Flap ${ }^{1}$

The principle of pressure adaptive honeycomb has been proven on the bench and in the wind tunnel. To enable a feasible design of this type of adaptive structure we need tools that can predict the behavior of this structure based on external loading and CDP. The objective is to develop these tools by relying on a finite element approximation of the pressurized honeycomb structure. Based on these tools we should be able to investigate the influence of certain design parameters, such as cell size, cell distribution and cell geometry on the effectiveness of a potential high lift device.

\section{APPLICATION: MORPHING FOWLER FLAP}

For high-subsonic transports the the Fowler motion of the flaps enhances lift in two ways: it increases the local wing chord and it increases the maximum lift coefficient through the jet-effect that is created by the slot between the main wing and the flap. The latter effect is a mutual beneficial interference effect that increases the lift coefficient of the wing substantially. The maximum lift coefficient is reached when flow separation starts on one of the wing components (slat, main wing, or flap). To get the highest lift coefficient, it is desired that stall occurs at each of these components simultaneously. In practice, however, this is difficult to achieve. However, by replacing the rigid flap by a variable-camber flap the separation can arguably be adjusted slightly and higher lift coefficients could be reached. This is schematically shown in Fig. 2. An additional benefit of variable camber is the possibility to influence the aft-loading of the wing and hence incrementally alter the aircraft pitching moment coefficient in order to reduce trim drag. This has been investigated by Airbus for many years ${ }^{2}$ but has not been implemented yet due to the complexity and weight of the associated kinematic system (a double-track mechanism was proposed).

Because the flap structure itself needs to carry bending and torsional loads in the present research it is proposed to leave this part of the structure intact and only change the geometry of the structure in front of the main spar and the structure behind the aft spar. In the proposed setup, these volumes are occupied with pressure-adaptive honeycomb which' pressure can be actively controlled. In Fig. 3 this is schematically shown. It is assumed that the morphing trailing edge of the flap measures $30 \%$ of the total flap chord. Two shapes are being identified in the present research. The first shape is that of the flap when no CDP is applied, but with the presence of external and internal loads. The external loads come from the pressure on the exterior of the flap, while the internal forces stem from the strained skin panels (see Section A. The second shape is found when a CDP is applied and the force field is still present. Given two prescribed shapes, the question is whether a topology of the pressure adaptive honeycomb and the skin can be found in order to morph between those shapes. 


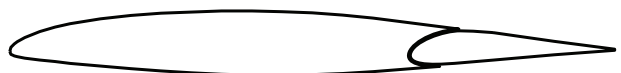

(a) cruise geometry

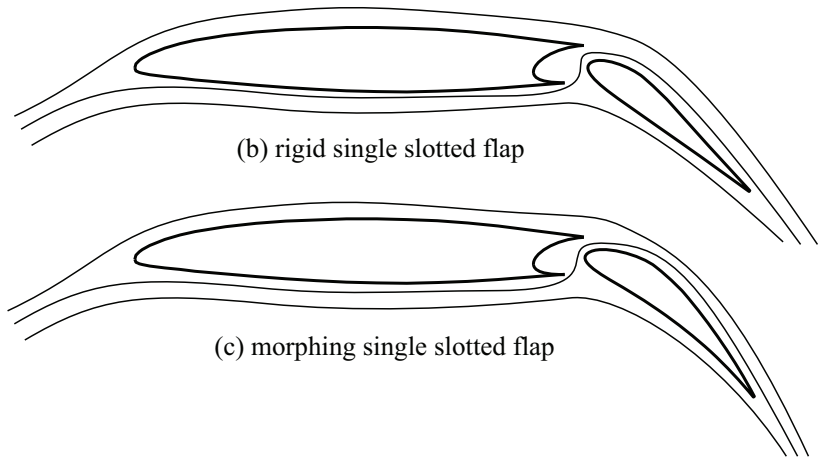

Figure 2. Flap Camber Increase Through Gross Geometrical Deformations to Increase Maximum Lift Coefficient.

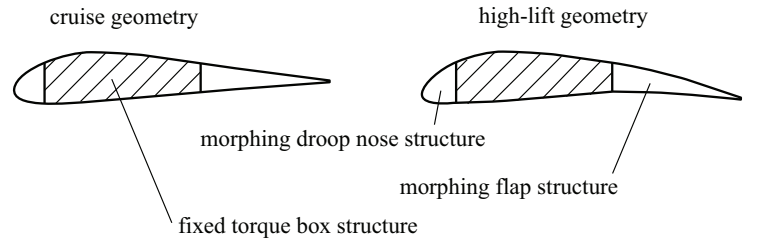

(a) Sketch of Structural Layout of the Morphing Flap in Cruise Geometry and High-Lift Geometry

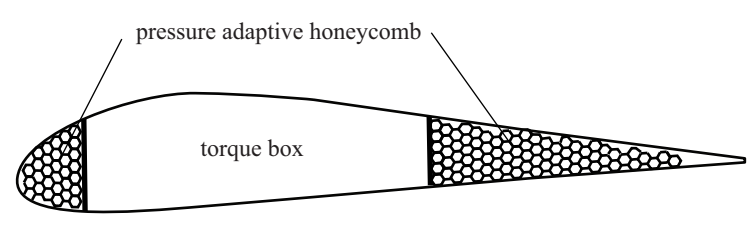

(b) Sketch of Pressure Adaptive Honeycomb Topology within Morphing Flap

Figure 3. Application of Pressure Adaptive Honeycomb in a Fowler Flap 


\section{A. Topology Design Methodology}

The goal of the present research is to develop a design tool for a pressure-adaptive flap as shown in Fig. 3 with a structural layout as was successfully demonstrated on the proof-of-concept test article (shown in Figure 1). The design tool should allow the user to design a morphing trailing edge where the skin thickness, the skin curvature, and the honeycomb topology can be altered such that a target shape in two different conditions can be found. These two conditions are the low-speed condition (target shape 1) and the highspeed condition (target shape 2). An schematic overview of this design tool is shown in Fig. 4 and is discussed in the following paragraphs.

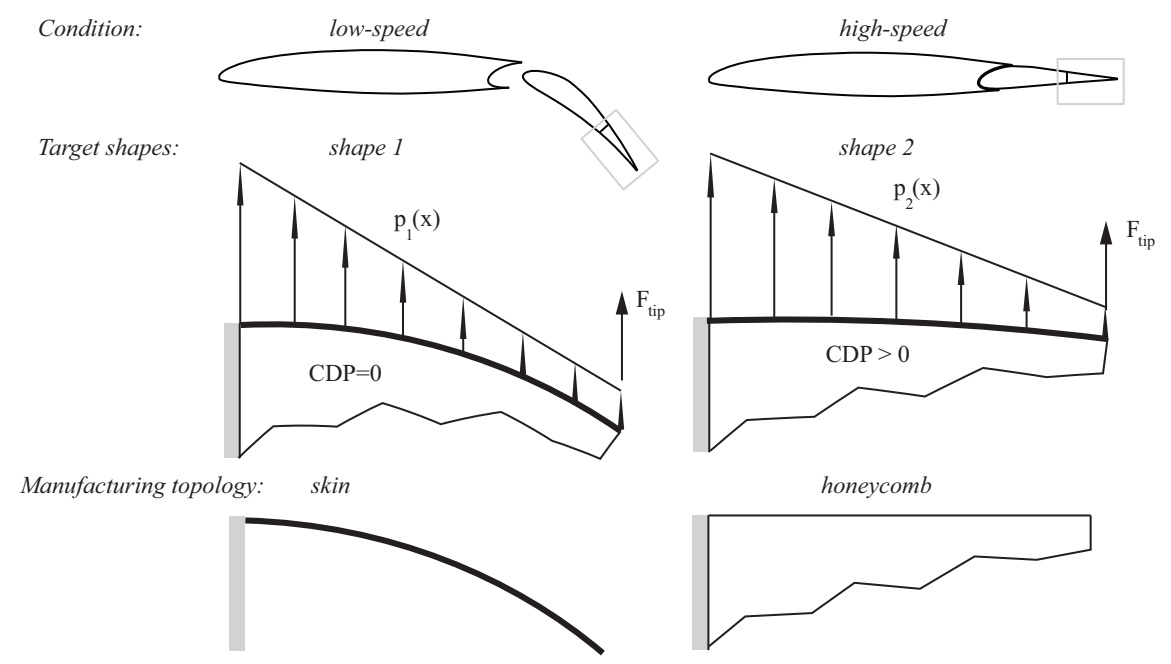

Figure 4. Sketch of Target Shapes and Load Cases

In the low speed condition, a target shape is provided that induces a large amount of morphing into the trailing edge of the flap. In this stage the CDP is assumed to be zero, or close to zero. The increase in camber is therefore solely introduced by the curved top skin. The aerodynamic loading in this condition counteracts the skin curvature and pulls the aft part of the the flap upwards. In the design tool it is determined what thickness and curvature distribution the skin should have when it is manufactured that it matches the target shape under the given aerodynamic load. This is done via an optimization loop where skin thickness and curvature are the design variables. The optimization loop stops whenever the calculated shape is sufficiently close to target shape 1 .

Once the skin thickness and curvature distribution are fixed, the next step is to find the honeycomb topology that allows the trailing edge to deform to the ideal shape in high-speed conditions (target shape 2 ). In this condition, we expect a target airfoil shape with much less trailing edge camber than in the lowspeed condition. To reduce the amount of aft camber it is therefore required to pressurize the honeycomb. In addition, a different aerodynamic load is present in this condition that should also be considered when optimizing the shape. In this optimization loop it is determined what the best CDP is to be applied to the honeycomb in the flap, such that it matches target shape 1 under a given aerodynamic load and at a given honeycomb topology.

In Fig. 5 the two consecutive optimization loops are shown. To calculate the shape of the trailing edge of the flap, the structure is divided in a finite number of bar elements that are connected by nodes. An internal finite element analysis is carried out to calculate the displacement, $d \bar{u}$, of each of the nodes. The displacement is a function of the overall structural stiffness, $\mathbf{K}$, and the residual forces, $\bar{r}$. The finite element method is further elaborated in Section III. The objective of the two optimization loops within the design tool is to present the desired manufacturing shape of both the skin and to determine the CDP of the honeycomb, such that a test article can be manufactured. 


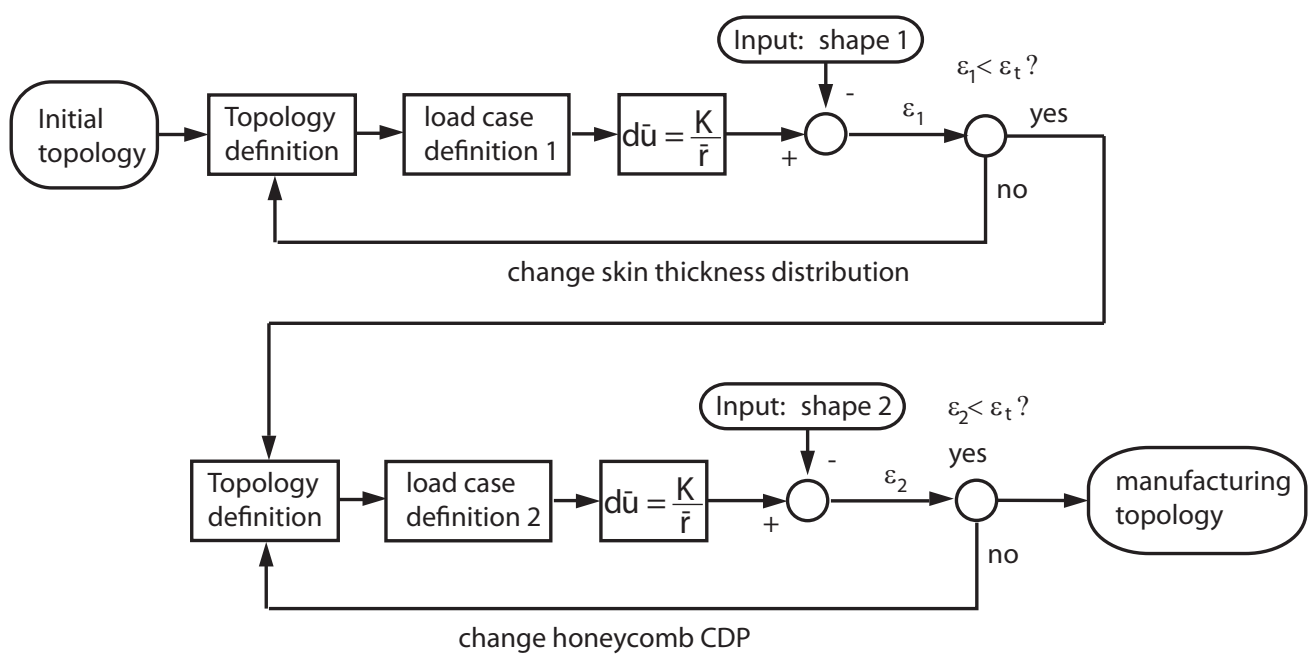

Figure 5. Flow Diagram of Topology Design Tool

\section{B. Generating Initial Topology}

The definition of the initial honeycomb topology within the flap volume is obtained in a series of steps. First of all, a rectangular honeycomb grid is obtained by defining the nodal coordinates and using simple geometric rules to connect them. The cell length, $l$ and cell wall angle, $\theta$ are input parameters for this process. Figure 6 shows these subsequent steps. The number of cells in the two orthogonal directions, as well as the characteristic honeycomb angle, $\theta$, can also be altered. In the optimization process, the values of $t / l$ and $\theta$ are important with respect to the deformation of the honeycomb and are therefore the two parameters that can be altered.

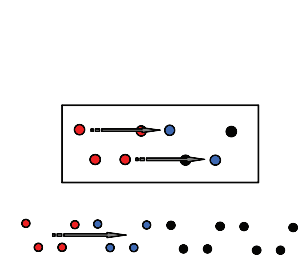

(a) Positioning of the first line

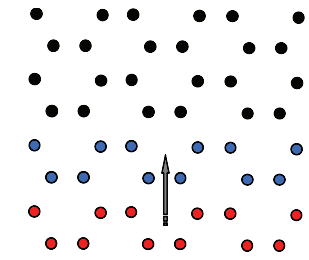

(b) Upwards translation

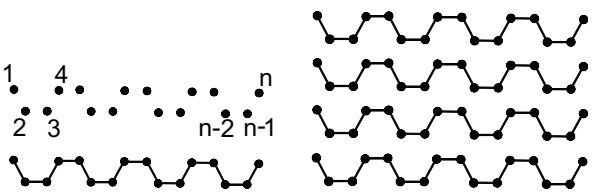

(c) Elements are con- (d) This repeated for nected from node 1 to each line node $n$

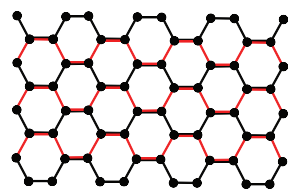

(e) Internal elements (red) are added similarly

Figure 6. Schematic representation of honeycomb generation algorithm

When the rectangular honeycomb grid is defined, the shape of the flap volume (without any external or internal load conditions) is projected onto the mesh. The boundary grid points are identified and displaced to the flap boundary. Grid points that are outside the flap boundary and are not attached to cells inside the boundary are discarded. The resulting shape is the initial topology, referred to in the flow diagram of Figure 5. This process is schematically shown in Figure 7. Note that the skin elements have different properties than the honeycomb elements.

\section{Aerodynamic Pressure Distribution}

The aerodynamic loading on the flap is one of the introduced external forces. The pressure at each node can be determined as long as the pressure at the root of the PAH section $\left(p_{1,2}(x=0)\right.$ in Figure 4$)$ is known. It is assumed that the difference in pressure between the upper and lower surface of the flap acts only on the upper surface. A triangular distribution is assumed for demonstration purposes. The only aerodynamic input parameter for the model is the pressure at the beginning of the morphing section of the flap. In the 


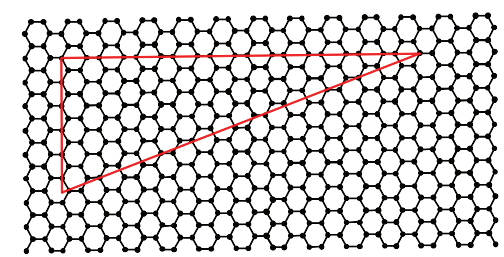

(a) Positioning the object in the honeycomb mesh

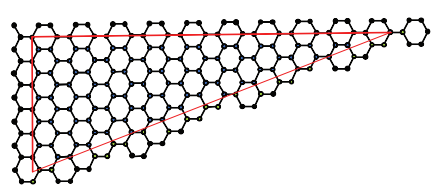

(b) Adding the margin and identifying the border nodes

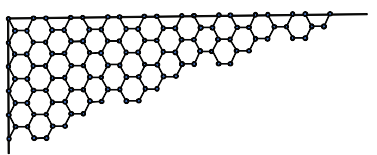

(c) The external nodes are connected to the boundary. Incomplete hexagons are deleted.

Figure 7. Volume meshing of a pressure adaptive flap

reference frame of the flap, this pressure occurs at $x=0$ and is denoted with $p(0)$. This value is calculated using:

$$
p(0)=\Delta C_{p}(x=0) q_{\infty}
$$

with $C_{p}(x=0)$ being the difference in pressure coefficient at $x=0$ and $q_{\infty}$ being the dynamic pressure. The values for $\Delta C_{p}$ can be deduced from experimental pressure distributions about airfoils.

To get a representative value for $C_{p}$ at $x=0$, data from a NACA report on a NACA 23012 airfoil with slotted flap is used. ${ }^{3}$ Table 1 gives an overview of all known data. From the pressure distribution of the airfoil with a 50 degree angle flap deflected, the $\Delta C_{p}$ is determined to be 1.1 (assumed PAH section length: $\left.0.3 c_{f}\right)$. This results in $p(0)_{\text {high-lift }}=862 \mathrm{~N} / \mathrm{m}^{2}$.

Table 1. Known data from NACA report $732,{ }^{3}$ for the calculation of the low speed pressure.

\begin{tabular}{|c|c|c|}
\hline Data & Value & Units \\
\hline Airfoil & NACA 23012 & - \\
$\alpha$ & 0 & degrees \\
$\delta_{f}$ & 50 & degrees \\
$\operatorname{Re}$ & $3 \mathrm{e} 6$ & - \\
$q_{\infty}$ & 16.37 & $\mathrm{lbs} / \mathrm{ft}^{2}$ \\
\hline
\end{tabular}

For the high-speed pressure, experimental data for a transonic supercritical airfoil is used. ${ }^{4}$ Table 2 provides all the appropriate data from the reference document. Again, $\Delta C_{p}$ is determined from the pressure distribution plot: $\Delta C_{p}=0.7$ (assumed PAH section length: $0.3 c_{f}$ ). The assumed cruise altitude is $35000 \mathrm{ft}$ resulting in $p(0)_{\text {cruise }}=6862 \mathrm{~N} / \mathrm{m}^{2}$.

Table 2. Known data for experimental testing of a transonic supercritical airfoil. ${ }^{4}$

\begin{tabular}{|c|c|c|}
\hline Data & Value & Units \\
\hline Airfoil & BAC-1 & - \\
$\alpha$ & 1 & degrees \\
$\delta_{f}$ & 0 & degrees \\
$\operatorname{Re}$ & $15 \mathrm{e} 6$ & - \\
$\mathrm{M}$ & 0.765 & - \\
\hline
\end{tabular}

Of course, these pressure values are not fully representative considering that different airfoils were used and $\Delta C_{p}$ is determined by estimating the position of the lines of the $C_{p}$ plot. However, for the moment, these values suffice as an indication of the expected aerodynamic pressure.

\section{Error Calculation and Convergence}

To determine the error between the target shape and the initial shape the skin is divided into a discrete number of panels, connected by nodes. The y-coordinates of the skin and geometry nodes are compared and 
their difference results in the error, $\varepsilon$. Figure 8 shows how this procedure works. By taking the halfwaypoint of two neighboring geometry nodes, the geometry is divided into compartments. If a skin node shares a compartment with a geometry node, they are matched as reference nodes. Geometry nodes without an assigned skin node are simply discarded as reference nodes. If more than one skin node can be assigned to a geometry node, only the closest one (y-direction) is assigned.

After processing the loadcases and subsequent deformation for both airfoils, the absolute distance is taken from the reference geometry node to its assigned skin node. In each of the optimization loops the calculated shape is compared to the target shape by evaluating the mean error between $N$ skin control points and the target control points:

$$
\varepsilon=\frac{1}{N} \sum_{i=1}^{N} \varepsilon_{i}
$$

The iterative process is said to have conversed when $\varepsilon<\varepsilon_{t}$. The optimization is stopped if the error falls below a given target error, $e_{t}$.

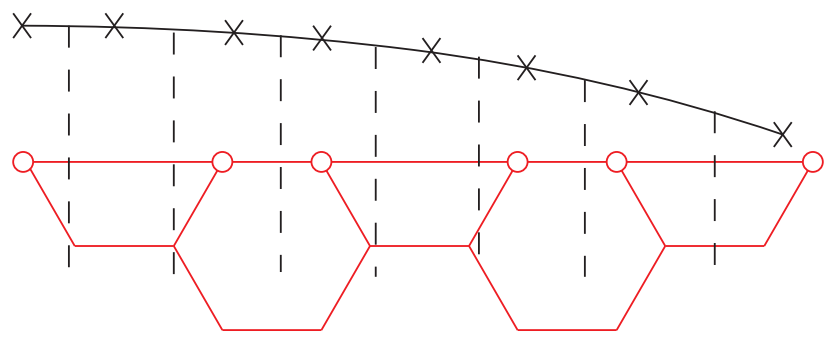

Figure 8. Procedure used to assign reference nodes. Skin nodes (red circles) are matched to their nearest geometry node (black crosses).

\section{THEORY OF THE FINITE ELEMENT MODEL}

The goal of the PAH Topology Optimization Tool is to design an internal flap geometry that possesses the capability to morph between two stable shapes. The actuation mechanism is the pressurization of that internal structure. From the work of $\operatorname{Vos}^{1}$ it is shown that honeycomb has very high strain properties in both longitudinal and lateral direction. These large structural deformations are the key principle behind PAH and this necessitates the use of nonlinear analysis. A very elegant approach for nonlinear structural FE analysis is provided by Felippa ${ }^{5}$ and will be described in more detail in the sections below.

\section{A. The Bar Element}

Honeycomb is a complex structure that is difficult to model because of the many interconnecting cell walls that make up the material. Since honeycomb is mainly used as core material for sandwich panels, the exact deformation of the individual cells is usually unimportant. For such cases, an often used method for modeling honeycomb in FE computations is to experimentally determine the prime material properties for a block of honeycomb material and apply these to a simple 'computational brick'. Obviously, for the purpose of PAH a more refined method is required as each individual cell must be analyzed in detail.

A more detailed approach is offered by looking into the micromechanical properties of the (repeating) honeycomb cells such as derived by Gibson. ${ }^{6}$ This gives an analytical derivation for cell wall bending. Extensions of this model are provided by Warren and Byskov ${ }^{7}$ (symmetry restrictions) and Park and Gao ${ }^{8}$ (combined bending and axial loading). However, all of the mentioned models are valid only for small angles and thus for small displacements. This makes them unfit for use in the proposed nonlinear model. Also, the analytical derivations require the analysis of many relations, which increases the complexity of the model and thus total computing time.

In order to keep the model as simple as possible, the current model is build up from Total Lagrangian (TL) bar elements. By using TL bars, the displacements of each individual bar can be calculated in a local reference frame and later converted to the global reference frame and added to the corresponding nodes. Due to the infinite bending stiffness of the bars, elongation is the only deformation possible. All bars are connected to nodes of the global honeycomb grid. These nodes are represented as frictionless hinges, thus 
bending stiffness is non-existing in the initial structure. Inherent stiffness is required in order to get a solution after deformation, so a small initial pressure differential is applied to the structure to simulate this. Figure 9 shows the used definition of a TL bar with respect to local and global coordinates.

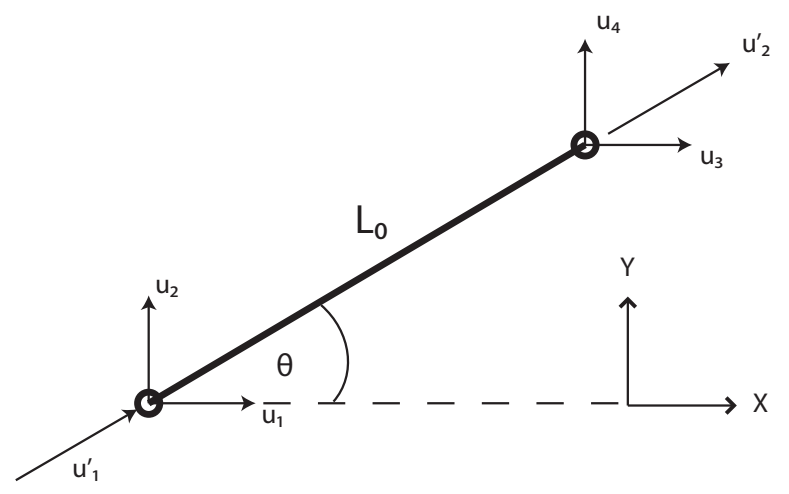

Figure 9. Generic TL bar element with displacements shown with respect to local and global coordinates.

\section{B. Applied Forces}

The mechanics of the FE model are directly related to the total energy of the entire system. This total energy consists of the internal (strain) energy potential $U$ and the external loads potential $V$ (Felippa ${ }^{5}$ ),

$$
\Pi=U-V
$$

Conservative forces that are active in the system can be determined by taking the first derivative of the potential functions,

$$
\bar{r}=\frac{\partial \Pi}{\partial \bar{u}}=\frac{\partial U}{\partial \bar{u}}-\frac{\partial V}{\partial \bar{u}}=\bar{p}-\bar{f}
$$

Nonconservative forces cannot be derived from a potential function and must therefore be derived directly from the applied forces. As can be seen from Equation 4 a distinction is made between internal forces and external forces. The derivation for each type of force is given below.

\section{Internal Forces}

The internal forces of the system are determined by the internal strain energy of the bars. Consider a tension force $N$ present in the generic bar. Figure 10(a) shows the components of the corresponding forces in the global coordinate system. Note that $\cos (\theta)=x_{21} / L$ and $\sin (\theta)=y_{21} / L$, where $x_{21}$ and $y_{21}$ follow from the definition in Figure 10(b),

$$
x_{21}=\left(X_{2}-X_{1}\right)+\left(u_{3}-u_{1}\right)
$$

and

$$
y_{21}=\left(Y_{2}-Y_{1}\right)+\left(u_{4}-u_{2}\right)
$$

Since the bar tension is triggered by the nodal displacements and the corresponding extension of the bar, the force components must be multiplied by the bar elongation factor $L / L_{0}$. Thus, the components of the tension induced forces become:

$$
\begin{gathered}
f_{x}=N \cos (\theta)=\frac{N}{L_{0}}\left[\left(X_{2}-X_{1}\right)+\left(u_{3}-u_{1}\right)\right] \\
f_{y}=N \sin (\theta)=\frac{N}{L_{0}}\left[\left(Y_{2}-Y_{1}\right)+\left(u_{4}-u_{2}\right)\right]
\end{gathered}
$$

The element force vector then becomes:

$$
\bar{p}=\frac{N}{L_{0}}\left[\begin{array}{c}
-\left(X_{2}-X_{1}\right)-\left(u_{3}-u_{1}\right) \\
-\left(Y_{2}-Y_{1}\right)-\left(u_{4}-u_{2}\right) \\
\left(X_{2}-X_{1}\right)+\left(u_{3}-u_{1}\right) \\
\left(Y_{2}-Y_{1}\right)+\left(u_{4}-u_{2}\right)
\end{array}\right]
$$




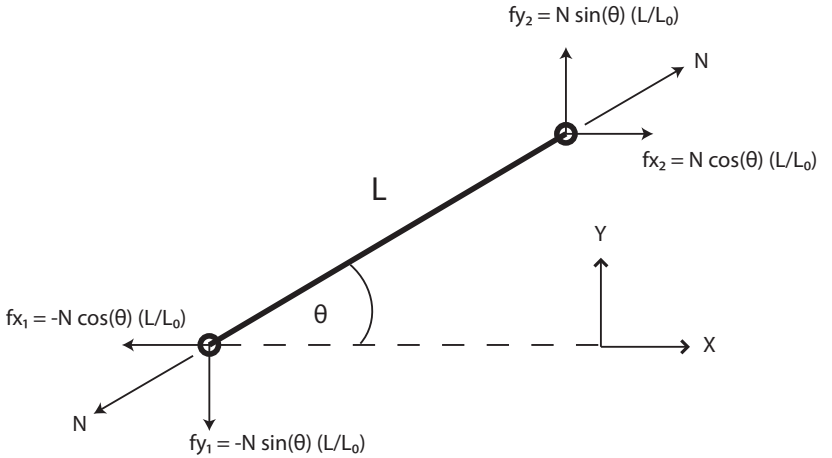

(a) force components as a result of a bar under tension.

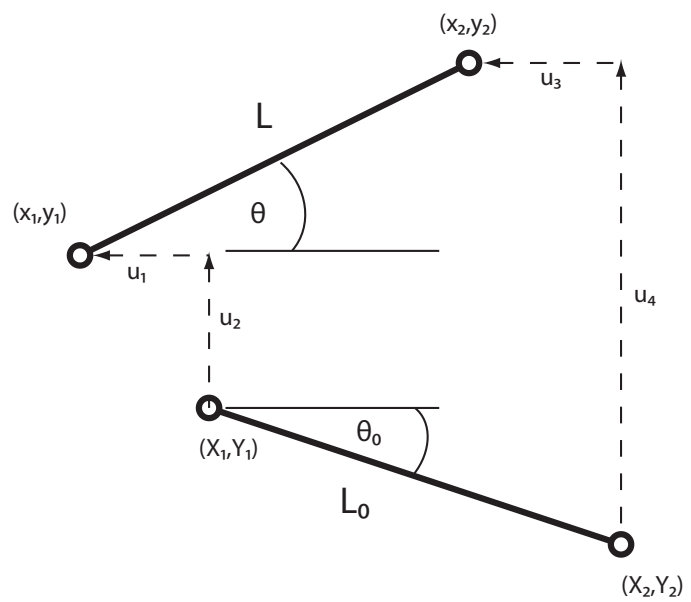

(b) coordinates and nodal displacements

Figure 10. Definition of nodal force and displacement components

\section{External Forces}

There are three external forces that may be applied on the system. The tool can apply a CDP to the cells, a simulated aerodynamic load on the top skin of the flap and/or a simulated 'bending skin' resistance force. All three of these force vectors are derived below.

The CDP loading acts as the actuator of the PAH and is therefor the main force applied on the structure. Also, since the structure itself possesses no inherent stiffness, a very small initial CDP is used to simulate structural rigidity when the structure is in a deflated state (zero CDP). Figure 11(a) shows the definition of a generic bar under a pressure load (direction of pressure is always perpendicular to the bar). The total force exerted on the bar is therefore: $f_{l}=p L$. This force is lumped on the connecting nodes of the bar and thus each node is loaded by an applied load of $p L / 2$ (a horizontal pressure distribution is assumed across the bar). The force components then become

$$
\begin{aligned}
f_{x} & =-\frac{1}{2} p L \sin \theta \\
f_{y} & =\frac{1}{2} p L \cos \theta
\end{aligned}
$$

or in vector format,

$$
\bar{f}_{\mathrm{CDP}}=\frac{1}{2} p L\left[\begin{array}{c}
-\sin \theta \\
\cos \theta \\
-\sin \theta \\
\cos \theta
\end{array}\right]
$$

From the geometry shown in Figure 10(b) it follows that $L \cos (\theta)=x_{2}-x_{1}=x_{21}$ and $L \sin (\theta)=y_{2}-y_{1}=y_{21}$. Substitution of Equations 5 and 6 into these relations and combining with Equation 12 gives

$$
\bar{f}_{\mathrm{CDP}}=\frac{1}{2} p\left[\begin{array}{c}
-\left(Y_{2}-Y_{1}\right)-\left(u_{4}-u_{2}\right) \\
\left(X_{2}-X_{1}\right)+\left(u_{3}-u_{1}\right) \\
-\left(Y_{2}-Y_{1}\right)-\left(u_{4}-u_{2}\right) \\
\left(X_{2}-X_{1}\right)+\left(u_{3}-u_{1}\right)
\end{array}\right]
$$

Figure 11(b) shows the definition of a generic bar under an aerodynamic load. Since it is assumed that the pressure distributed across the flap has a triangular shape, the pressure at each node of the element must be determined. It is further assumed that the pressure reduces linearly towards the tip of the flap (Figure 4). Since the trailing edge of the flap is likely not to contain any pressure-adaptive honeycomb, the aerodynamic pressure load is lumped into a single tip force, $F_{\text {tip }}$ (see Fig. 4). As with the CDP loading, the 


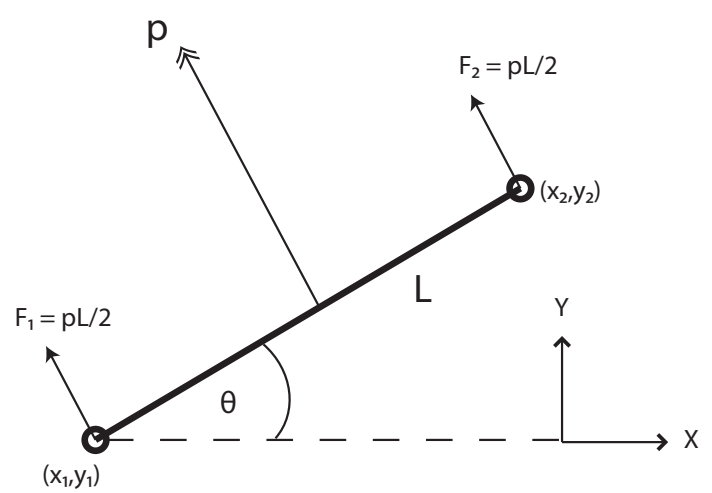

(a) Bar element under pressure loading

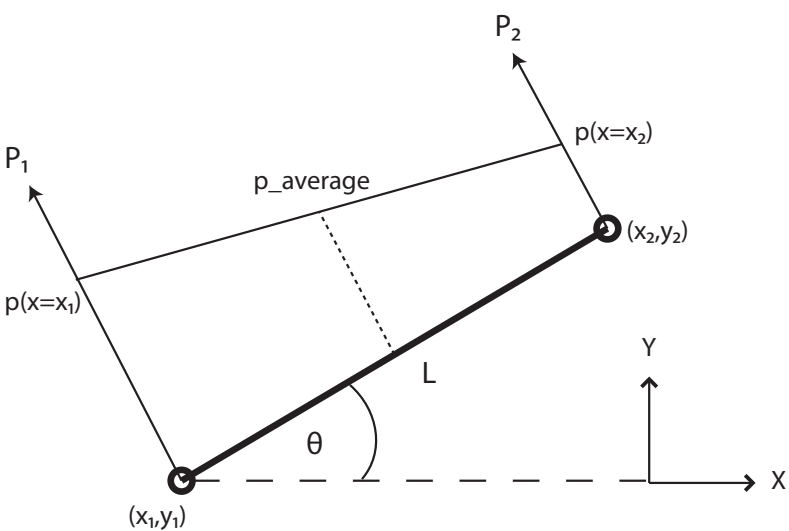

(b) Bar element under aerodynamic loading

Figure 11. Loads introduced through pressure

force is lumped on the connecting nodes of the bar and thus each node is loaded by an applied load of $P_{1}$ or $P_{2}$. This is done by determining the pressure at the bar coordinates $x_{1}$ and $x_{2}$.

$$
\begin{aligned}
& p_{\left(x=x_{1}\right)}=p_{(x=0)} \frac{L_{\text {skin }}-x_{1}}{L_{\text {skin }}} \\
& p_{\left(x=x_{2}\right)}=p_{(x=0)} \frac{L_{\text {skin }}-x_{2}}{L_{\text {skin }}}
\end{aligned}
$$

where $L_{\text {skin }}$ is the total length of the skin across the PAH section and the tip. The average pressure across the bar (the pressure at the midsection) is then:

$$
p_{\mathrm{av}}=\frac{p_{x_{1}}+p_{x_{2}}}{2}
$$

The lumped forces for each node are then calculated using the average pressure at each half of the bar

$$
\begin{aligned}
& P_{1}=\frac{p_{x_{1}}+p_{\text {av }}}{2} \frac{L}{2} \\
& P_{2}=\frac{p_{x_{2}}+p_{\text {av }}}{2} \frac{L}{2}
\end{aligned}
$$

The force vector then becomes

$$
\bar{f}_{\text {aero }}=\frac{1}{L}\left[\begin{array}{c}
-P_{1}\left[\left(Y_{2}-Y_{1}\right)+\left(u_{4}-u_{2}\right)\right] \\
P_{1}\left[\left(X_{2}-X_{1}\right)+\left(u_{3}-u_{1}\right)\right] \\
-P_{2}\left[\left(Y_{2}-Y_{1}\right)+\left(u_{4}-u_{2}\right)\right] \\
P_{2}\left[\left(X_{2}-X_{1}\right)+\left(u_{3}-u_{1}\right)\right]
\end{array}\right]
$$

The final loadcase is a simulated skin bending resistance. Since the bar elements are connected through frictionless hinges, bending moments are not taken into account. This causes an inconsistency compared to 'real life' on the influence of an aluminum skin covering the upper side of the flap. To simulate skin bending behavior, an equivalent (correction) force is introduced through the analysis of the rotational angle of the bar with respect to the global axes (see Figure 12(a)). It is assumed that on each skin node (except for the very last) a torsion spring is present with a spring stiffness based on an aluminum bar according to the theory of material mechanics from Gere. ${ }^{9}$ Thus,

$$
k_{\text {rot }}=\frac{3 E I}{L^{3}}
$$


where

$$
I=\frac{1}{12} L t^{3}
$$

For a given material stiffness, $E$, and bar lenght, $L$, Eq. 20 links the spring stiffness to the plate thickness, $t$. The moment $M_{\text {rot }}$ is then dependent on the difference between the angle $\varphi$ and the target angle $\varphi_{0}$ as shown in Figure 12(b). The target angle $\varphi_{0}$ is implicitly defined by the target shape of the morphing flap (shape 1), which can be translated to a top-skin curvature distribution. This curvature distribution, in turn, is translated to a discrete set of target rotations of the bars, $\varphi_{0}$. Angle $\varphi$ is calculated using $\theta_{\text {rot }}$,

$$
\varphi_{i}=\pi-\theta_{\text {rot }}(i)+\theta_{\text {rot }}(i-1)
$$

and therefore:

$$
M_{\text {rot }}=k_{\text {rot }}\left(\varphi_{0}-\varphi\right)
$$

which results in a correction force $F_{\text {eq }}$ to force the skin in the direction of the target shape.

$$
F_{\text {eq }}=\left|\frac{M_{\text {rot }}}{L}\right|
$$

Since the bending moment can be either positive or negative depending on the rotational angle of the bar with respect to its neighbor, there are two variants of the force vector. For brevity, only the positive bending scenario is derived. In this case the force components become

$$
\begin{aligned}
& f_{x}=-F_{\text {eq }} \sin \theta \text { and } \\
& f_{y}=F_{\text {eq }} \cos \theta
\end{aligned}
$$

In case of a negative moment, the signs of the equations change. In vector form for a bar this results in

$$
\bar{f}_{\text {skin }}=\frac{F_{\text {eq }}}{L}\left[\begin{array}{c}
0 \\
0 \\
-\left(Y_{2}-Y_{1}\right)-\left(u_{4}-u_{2}\right) \\
\left(X_{2}-X_{1}\right)+\left(u_{3}-u_{1}\right)
\end{array}\right]
$$

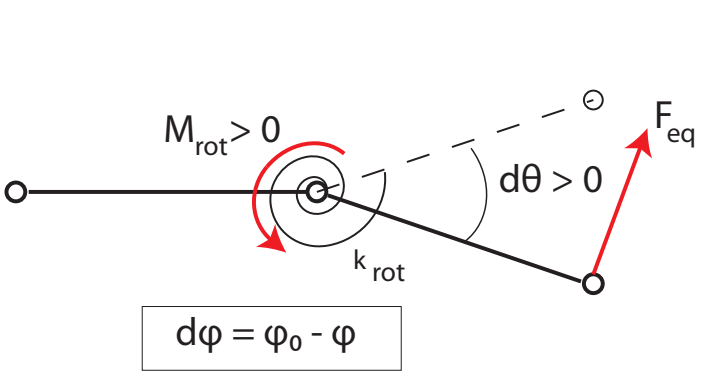

(a) Force, $F_{\text {eq }}$ is introduced through the moment, $M_{\text {rot }}$

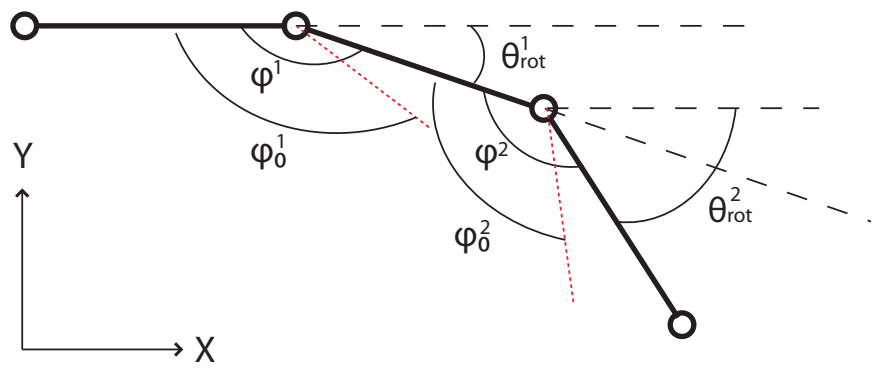

(b) Definition of bar angles in during bending

Figure 12. Loads introduced through skin bending

\section{Stiffness Matrices}

From each bar a total of three stiffness matrices are derived. These matrices are known as the material stiffness matrix $\left(\mathbf{k}_{m}\right)$, the geometric stiffness matrix $\left(\mathbf{k}_{g}\right)$ and the load stiffness matrix $\left(\mathbf{k}_{l}\right)$. Given that a $\mathrm{FE}$ bar has four degrees-of-freedom, these three matrices each are of size $4 \times 4$. The local stiffness matrices are then assembled into the global stiffness matrices $\mathbf{K}_{m}, \mathbf{K}_{g}$ and $\mathbf{K}_{l}$ and subsequently combined into a single global stiffness matrix $\mathbf{K}$. Matrix $\mathbf{K}$ contains all inherent stiffness properties of the structure.

$$
\mathbf{K}=\mathbf{K}_{m}+\mathbf{K}_{g}+\mathbf{K}_{l}
$$


These stiffness matrices are directly derived from the potential functions or the forces, thus

$$
\mathbf{K}=\frac{\partial^{2} \Pi}{\partial \bar{u}^{2}}=\frac{\partial^{2} U}{\partial \bar{u}^{2}}-\frac{\partial^{2} V}{\partial \bar{u}^{2}}=\frac{\partial \bar{p}}{\partial \bar{u}}-\frac{\partial \bar{f}}{\partial \bar{u}}
$$

where

$$
\frac{\partial \bar{p}}{\partial \bar{u}}=\mathbf{K}_{m}+\mathbf{K}_{g}
$$

and

$$
-\frac{\partial \bar{f}}{\partial \bar{u}}=\mathbf{K}_{l}
$$

The following subsections provide the derivations of the individual stiffness matrices. It should be noted that stiffness matrix $\mathbf{K}_{l}$ is a combination of all external loadcases applied on the model, thus

$$
\mathbf{K}_{l}=\mathbf{K}_{\mathrm{CDP}}+\mathbf{K}_{\mathrm{aero}}+\mathbf{K}_{\mathrm{skin}}
$$

This is explained in more detail in Section 3.

\section{Material Stiffness Matrix}

The bar displacements in both local and global coordinates are given as in Figure 9. Following the derivation by Ferreira, ${ }^{10}$ the global stiffness matrix for the generic bar is given.

The local coordinates are given as

$$
\bar{u}^{T}=\left[u_{1}^{\prime} u_{2}^{\prime}\right]
$$

and the global coordinates as

$$
\bar{u}^{T}=\left[\begin{array}{llll}
u_{1} & u_{2} & u_{3} & u_{4}
\end{array}\right]
$$

The relation between the two reference frames depends on the angle $\theta$ and can be written as

$$
\begin{aligned}
& u_{1}^{\prime}=u_{1} \cos (\theta)+u_{2} \sin (\theta) \\
& u_{2}^{\prime}=u_{3} \cos (\theta)+u_{4} \sin (\theta)
\end{aligned}
$$

Rewriting above equations in matrix form gives

$$
\bar{u}^{\prime}=\mathbf{L} \bar{u}
$$

with $\mathbf{L}$ the transformation matrix defined as

$$
\mathbf{L}=\left[\begin{array}{llll}
c & s & 0 & 0 \\
0 & 0 & c & s
\end{array}\right]
$$

where $c=\cos \theta=X_{21} / L_{0}$ and $s=\sin \theta=Y_{21} / L_{0}$. The stiffness matrix in the local coordinate system is given by the stiffness of the bar (axial deformation only)

$$
\mathbf{k}_{m}=\frac{E A}{L_{0}}\left[\begin{array}{cc}
1 & -1 \\
-1 & 1
\end{array}\right]
$$

To convert this stiffness matrix from local coordinates to global coordinates, $\mathbf{k}_{m}$ must be multiplied with the transformation matrix introduced in Equation 38

$$
\mathbf{K}_{m}=\mathbf{L}^{T} \mathbf{k}_{m} \mathbf{L}
$$

which becomes

$$
\mathbf{K}_{m}=\frac{E A}{L_{0}}\left[\begin{array}{cccc}
c^{2} & c s & -c^{2} & -c s \\
c s & s^{2} & -c s & -s^{2} \\
-c^{2} & -c s & c^{2} & c s \\
-c s & -s^{2} & c s & s^{2}
\end{array}\right]
$$

Note that $\mathbf{K}_{m}$ only depends on the material properties and not directly to the internal (strain) force. Therefore it would not show up when directly derived from the internal force $\bar{p}$ as in Equation 30 . 


\section{Geometric Stiffness Matrix}

The geometric stiffness matrix accounts for the element deformation which occurs when considering finite displacements. It is based on the analysis of the tension in the bars. The element geometric stiffness matrix in global coordinates is then the first derivative of the internal (strain) force vector to $\bar{u}$ and thus becomes

$$
\mathbf{K}_{g}=\frac{\partial \bar{p}}{\partial \bar{u}}=\frac{N}{L_{0}}\left[\begin{array}{cccc}
1 & 0 & -1 & 0 \\
0 & 1 & 0 & -1 \\
-1 & 0 & 1 & 0 \\
0 & -1 & 0 & 1
\end{array}\right]
$$

\section{Load Stiffness Matrix}

The load stiffness matrix contains another load induced stiffness like the geometric stiffness. It contains three distinct loadcases of which the force vectors have already been derived in Section 2, but the second derivative of the applied energy is the contribution to the global stiffness matrix $\mathbf{K}$. The element load stiffness matrix in global coordinates is then the negative of the first derivative of the load force vector $\bar{f}$ to $\bar{u}$ (Equation 31). For the CDP load case this becomes:

$$
\mathbf{K}_{\mathrm{CDP}}=-\frac{\partial \bar{f}_{\mathrm{CDP}}}{\partial \bar{u}}=\frac{1}{2} p\left[\begin{array}{cccc}
0 & -1 & 0 & 1 \\
1 & 0 & -1 & 0 \\
0 & -1 & 0 & 1 \\
1 & 0 & -1 & 0
\end{array}\right]
$$

Beside the CDP loading, there are two more model specific loadcases. These are the aerodynamic loading and the skin bending. Their corresponding force vectors have also been derived in Section 2. As with the CDP loadcase, the negative derivative of these vectors give the contribution to the global stiffness matrix. For the aerodynamic loadcase,

$$
\mathbf{K}_{\text {aero }}=-\frac{\partial \bar{f}_{\text {aero }}}{\partial \bar{u}}=\frac{1}{L}\left[\begin{array}{cccc}
0 & -P_{1} & 0 & P_{1} \\
P_{1} & 0 & -P_{1} & 0 \\
0 & -P_{2} & 0 & P_{2} \\
P_{2} & 0 & -P_{2} & 0
\end{array}\right]
$$

For the skin bending loadcase, there are two different scenarios depending on the sign of the bending moment as described in Section 2. For a positive bending moment, the skin bending stiffness matrix becomes

$$
\mathbf{K}_{\text {skin }}=-\frac{\partial \bar{f}_{\text {skin }}}{\partial \bar{u}}=\frac{F_{\text {eq }}}{L}\left[\begin{array}{cccc}
0 & 0 & 0 & 0 \\
0 & 0 & 0 & 0 \\
0 & -1 & 0 & 1 \\
1 & 0 & -1 & 0
\end{array}\right]
$$

For a negative bending moment the matrix elements simply change sign.

\section{Finite Element Solution}

When the global stiffness matrix $\mathbf{K}$ and the force vector $\bar{r}$ are compiled, the relative displacements of each node is calculated using

$$
d \bar{u}=\frac{\mathbf{K}}{\bar{r}}
$$

These displacements are then added to the total displacement with respect to the reference (original) coordinates: $\bar{u}=\bar{u}+d \bar{u}$. Based on the elongations of the bars and the resulting stresses, a new force vector and corresponding global stiffness matrix is derived. This iterative process continues until the difference in largest nodal force (with respect to the previous iteration) is smaller than 1e-10. At this point the solution is assumed to have converged to a stable position. 


\section{MODEL VALIDATION}

In this section the finite-element model of Section III is validated against an analytical model and against experimental tests. The main reason for this, is to prove that a model constructed with the proposed finite element method generates results that are sufficiently accurate and can thus be used in a more extensive and complex PAH design tool. To demonstrate the nonlinear behavior of the model, an analytical derivation of the deformation of a pressurized quarter hexagon is compared to the numerical result for a single hexagonal cell in Section A. In Section B, the numerical results are compared against experimental data of a compressionextension test of a single pressurized honeycomb cell.

\section{A. Comparison with Analytic Theory}

To prove the validity of the model for finite displacements, an analytical model is derived for the deformation of a quarter hexagon for a varying load. Because of the double symmetric shape of the hexagon cell, a quarter hexagon is sufficiently accurate for this purpose. Figure 13 shows the schematic representation of the quarter hexagon.

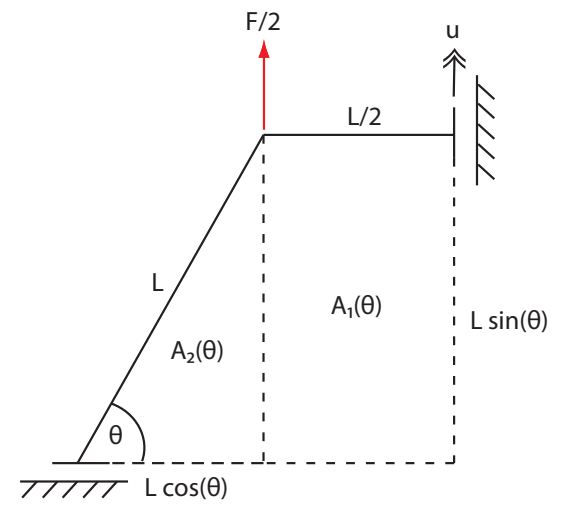

Figure 13. Quarter hexagon definition used for the derivation of an analytical approximation of the nonlinear model.

From theory it is known that the total energy of the system is equal to the internal energy $-p V$ plus the externally applied work $F u$. Since this is a 2-dimensional problem, the volume can be replaced by the area, which is a function of the cell wall angle $\theta$.

$$
\Pi=-p A(\theta)+\frac{F}{2} u(\theta)
$$

From geometry, the following relations for the area can be derived,

$$
\begin{gathered}
A_{1}(\theta)=\frac{L^{2}}{2} \sin \theta \\
A_{2}(\theta)=\frac{L^{2}}{2} \sin \theta \cos \theta
\end{gathered}
$$

Combining Equations 48 and 49 gives the relation for the total area as a function of $\theta$,

$$
A(\theta)=\frac{L^{2}}{2} \sin \theta(1+\cos \theta)
$$

The displacement $u$ is also described as a function of $\theta$.

$$
u(\theta)=L \sin \theta-L \sin \theta_{i}
$$

where $\theta_{i}$ is the initial (neutral) angle of 60 degrees. The next step is to minimize the total system energy, such that the two energy terms can be compared in the system equilibrium position.

$$
\frac{\partial \Pi}{\partial \theta}=-\frac{p L^{2}}{2}\left(\cos \theta+\cos ^{2} \theta-\sin ^{2} \theta\right)+\frac{F}{2} L \cos \theta=0
$$


From Equation 52 a function of $F(\theta)$ can be derived.

$$
F(\theta)=\frac{p L}{\cos \theta}\left(\cos \theta+\cos ^{2} \theta-\sin ^{2} \theta\right)
$$

Now that both force and displacement are derived as a function of $\theta$, a plot of the honeycomb deformation behavior can be made and compared to the results of the analytical model. Note that $u(\theta)$ as derived in Equation 51 is the displacement for the top half of the cell only. This value should be doubled to be representative for the numerical model. Figure 14 shows a plot of the numerical and analytical deformation analysis of a honeycomb cell for pressure $p=41 \mathrm{kPa}$ (ambient pressure assumed zero, thus CDP $=41 \mathrm{kPa}$ ) and cell wall length $L=20 \mathrm{~mm}$. Based on the results of this plot it seems safe to assume that the numerical model is also sufficiently accurate at large deformations.

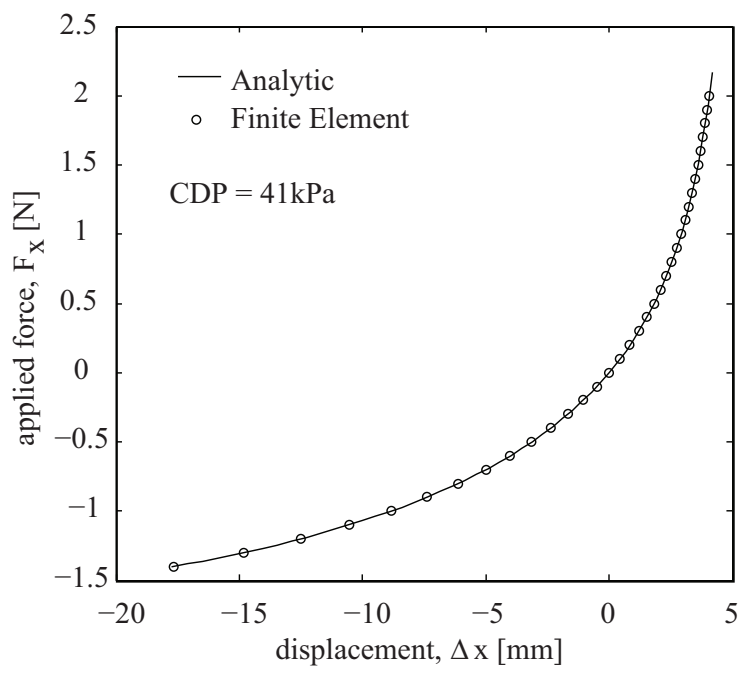

Figure 14. Comparison between the analytical and numerical results.

\section{B. Comparison with Experimental Data}

In the previous subsection, the accuracy of the numerical model has been proven with respect to theory. How this compares to experimental results is demonstrated in the proceeding paragraphs. Vos ${ }^{1}$ performed a compression-extension experiment with a single pressurized honeycomb cell with the same dimensions as in Figure 14. In this subsection, these experimental results are compared against those of the numerical model for a single cell.

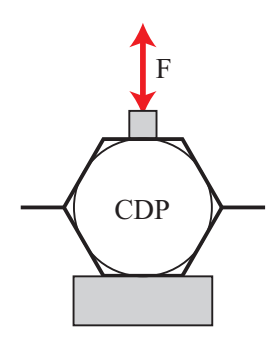

Lateral

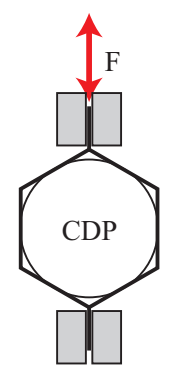

Longitudinal

Figure 15. A graphical representation of the lateral and longitudinal deformation experiments.

Figure 15 shows a simplified representation of the experimental setup. The exact experimental setup is described in detail in the referred document ${ }^{1}$ and will not be discussed here. A honeycomb cell was deformed in both lateral and longitudinal direction for various CDP values. Since the numerical model uses a small initial pressure $(\mathrm{CDP}=1 \mathrm{kPa})$ to provide structural rigidity at the deflated state, a small inconsistency 
exists with the experimental results. This error is amplified at lower values for CDP as the initial pressure will be relatively high. It is therefore assumed that this error becomes negligible at higher CDP values and since $41 \mathrm{kPa}$ was the largest applied CDP during the experiments, this was taken as a reference value for the numerical model. Figures 16(a) and 16(b) show the force-displacement relation as measured during the experiments and predicted by the numerical simulations.

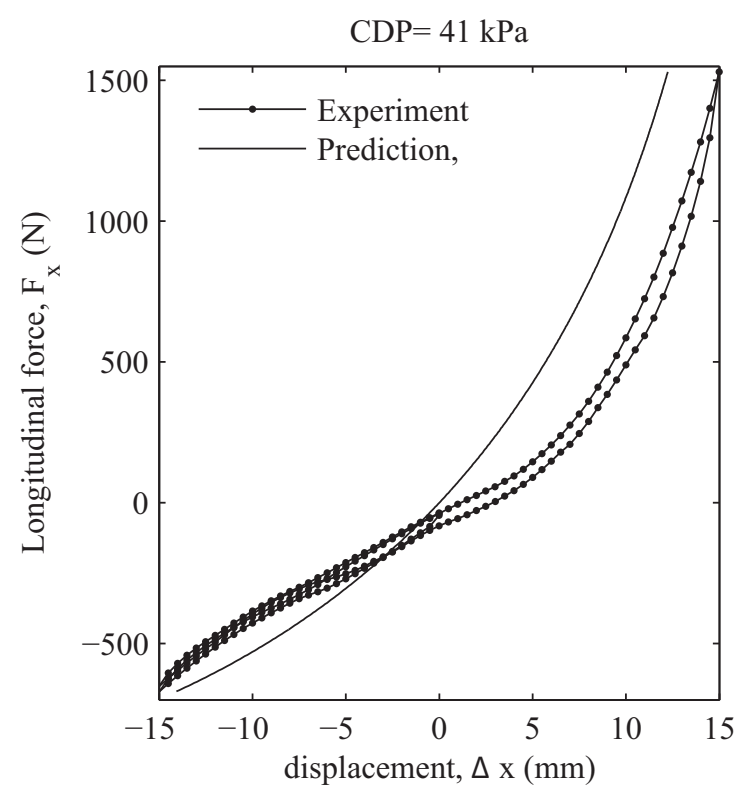

(a) Longitudinal force-displacement plot

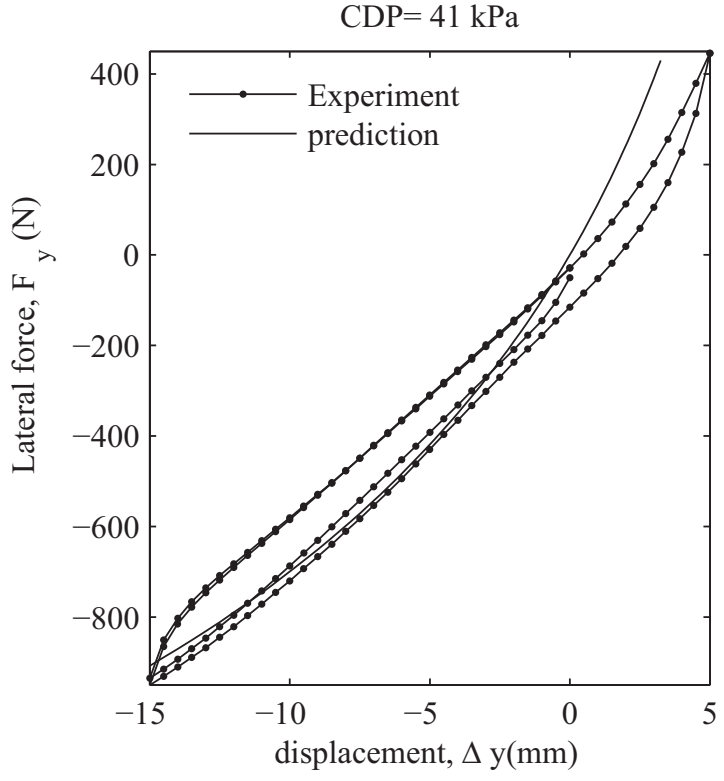

(b) Lateral force-displacement plot

It appears that the numerical results show a better correlation with the lateral deformation than with the longitudinal deformation. The largest inconsistencies are found at the edges of the plot, where deformation is largest. A possible explanation could be that the flattening effect of the physical hexagonal structure is slowing the displacement when nearing its maximum. At this point, the pouch containing the pressurized air is being compressed to its minimum volume. The numerical model does not consider a pouch being present inside the cell, but simply simulates this pouch pressure by adding its contribution to the cell wall element directly. As a result, the hexagonal cell can be compressed to zero degrees cell wall angle or even to negative angles since no boundaries have been defined. In order to get a closer match with the longitudinal deformation, the numerical model should be corrected for the reduced pressurized volume due to the pouch. It can be shown that the stiffness reduction due to this effect is proportional to the volume of the pouch with respect to the volume of the honeycomb cell. In the ideal case where the pouch forms a perfect circle inside a perfect hexagon, the volumetric ratio amounts to $0.91 .^{1}$

\section{DEMONSTRATION OF TOPOLOGY DESIGN TOOL}

This section discusses the applicability of the tool based on a number of examples. Table 3 shows a list of the performed tests and their input parameters. The length $l$ is the cell wall length and $H$ the total height at the root of the PAH section as defined in Fig. 16. Table 3 also shows the distribution of $\varphi_{0}$ along the skin panels. This angle is defined in Fig. 12(b) and can be linked to the curvature of skin when it is manufactured. For these specific tests, the $\varphi_{0}$ distribution was kept constant at $0.99 \pi$ at all skin nodes except for the angle at node $1\left(\varphi_{0}^{1}\right)$, which was varied for each test. The first element differs from the rest because of a larger starting angle. Finally, the CDP is shown as an input for the demonstration tool. At the time of submission of this paper, the second optimization loop in Fig. ?? was not yet operational. Therefore, only the skin thickness topology (result of loop 1) was generated as output. In the near future, the tool is modified, such that also the CDP can be obtained as an output for shape 2 . 
Table 3. Input variables for tool demonstration

\begin{tabular}{cccccccc}
\hline Test \# & $l[\mathrm{~mm}]$ & $H[\mathrm{~mm}]$ & Chord-tip & $\mathrm{CDP}_{\text {shape } 1}[\mathrm{kPa}]$ & $\mathrm{CDP}_{\text {shape } 2}[\mathrm{kPa}]$ & $\varphi_{0}^{1}[\mathrm{rad}]$ & $\varphi_{0}^{i} \forall i \backslash 1[\mathrm{rad}]$ \\
\hline 1 & 10 & 80 & $0.2-0.1$ & 3 & 100 & $0.90 \pi$ & $0.99 \pi$ \\
2 & 8 & 100 & $0.2-0.1$ & 6 & 100 & $0.90 \pi$ & $0.99 \pi$ \\
3 & 22 & 120 & $0.3-0.15$ & 2 & 100 & $0.88 \pi$ & $0.99 \pi$ \\
4 & 8 & 80 & $0.2-0.05$ & 7 & 100 & $0.90 \pi$ & $0.99 \pi$ \\
\hline
\end{tabular}

\section{A. Interpreting the Tool Output}

The output of the tool is a plot similar to the one shown in Figure 17. It presents the high-speed and the low-speed shapes. In addition, the target shapes are presented such that one can compare how well the target shape is approached in both conditions. In all examples the same target airfoils are employed: a NACA 2412 for the cruise shape and a NACA 9412 for the high-lift shape. Only the aft $30 \%$ of the chord is flexible and contains the PAH core.

The axes of the plot show the position of the structure in millimeters. It is assumed that the $\mathrm{x}$-axis is parallel to the flap chord. The origin is set at the top left of the connection of the PAH to the fixed structure of the flap. To size the PAH, a few parameters are required at the input section of the program. The three key sizing parameters are: Flap Chord, PAH Chord and Solid Tip. These are also graphically portrayed in Figure 16. For example, in the first test, the PAH Chord is set to $0.2 c_{f}$ and the Solid Tip section is set to $0.1 c_{f}$. In all tests, the Flap Chord is fixed at $1000 \mathrm{~mm}$. For the first example, the deforming part of the flap measures $200 \mathrm{~mm}$, while the length over which the honeycomb is applied measures $100 \mathrm{~mm}$.

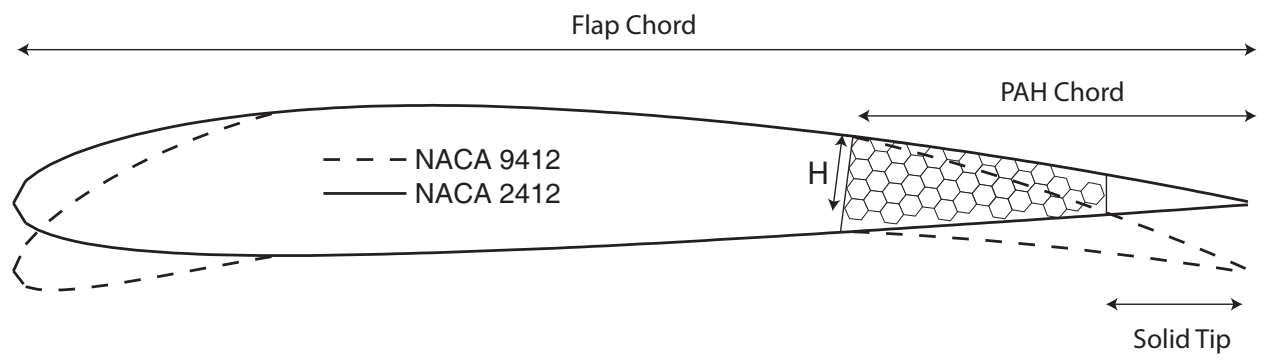

Figure 16. Three main sizing parameters for determining the size of the PAH section.

\section{B. Discussion of Demonstration Results}

The first test is a relatively compact PAH section with a small number of cells (Figure 17). Both calculated shapes lie very close to the target shapes and it should therefore be possible for such a structure to deform from one position to the other. Note that the truss structure at the end of the PAH represents the solid tip. In this design, the PAH is connected to this tip, as well as to the root of the section. This is analogous to the structure in the prototype test article of Fig. 1.

The second test considers the same section, but with smaller (and therefore more) cells. The PAH section is also slightly higher at the root $(H=100)$, which results in a slightly thicker section. The results shown in Figure 18 show that the solution is almost similar to that of test number 1. Both shapes match their target shapes closely. Based on these findings it seems that the size of the cells does not make much difference. The maximum deformation is limited by the amount of material that can move freely. Whether this 'free' material is distributed across many small cells or only a few large cells makes no difference to the result.

Test 3 shows what happens when the same PAH section consists of only a few (5) very large cells. The match between calculated and target shape is now less accurate (Figure 19). This is due to the coarse node distribution. With such large cells, there is only a very small number of reference nodes to determine the error from. Another problem is the truss section at the cut-off point where the tip begins. This very stiff section causes a boundary error that is amplified at very small numbers of cells. 


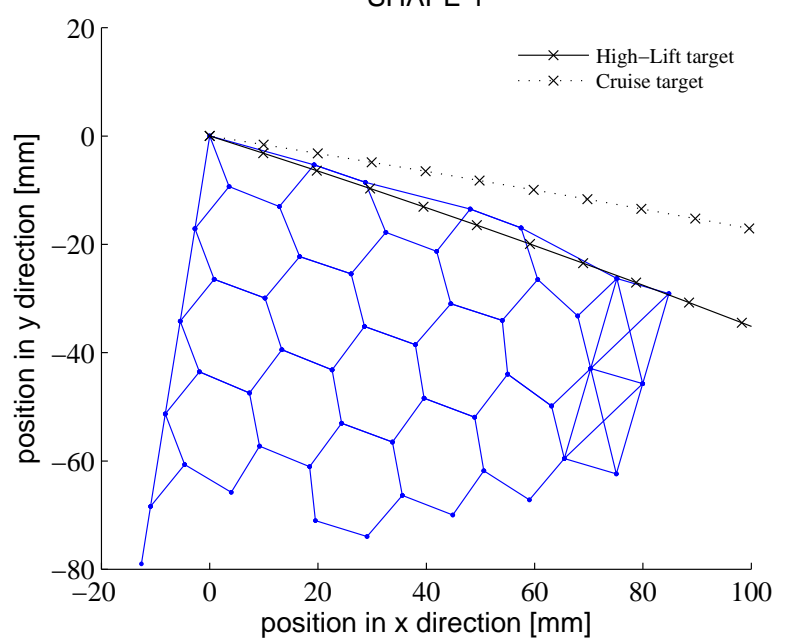

(a) High-lift position

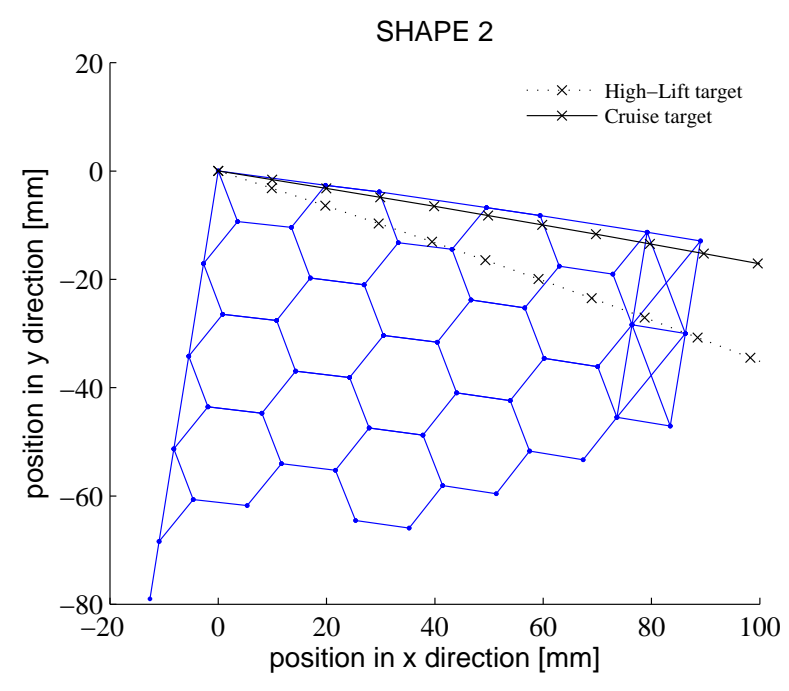

(b) Cruise position

Figure 17. Results of test \# 1 .

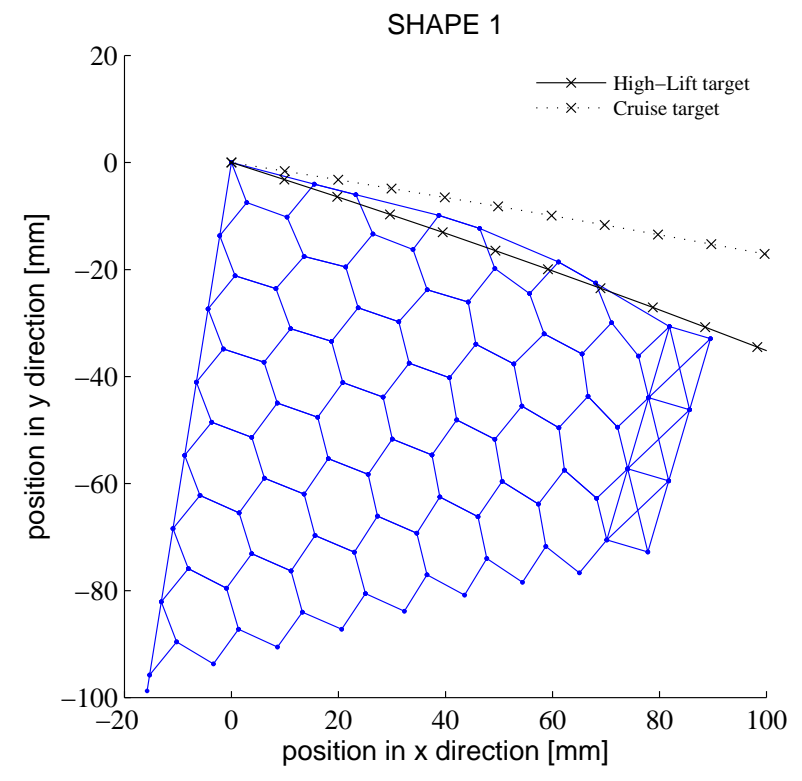

(a) High-lift position

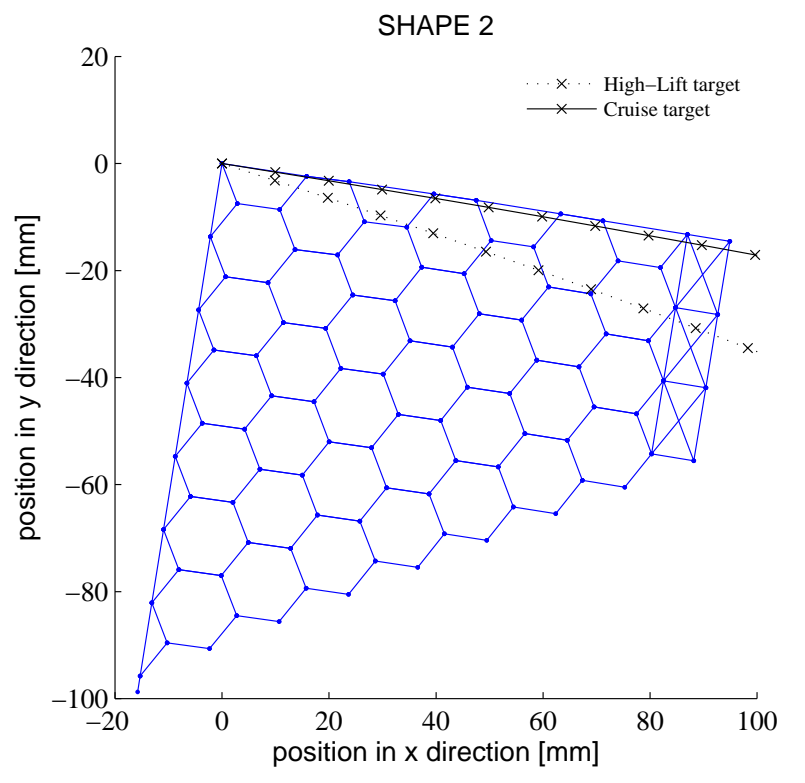

(b) Cruise position

Figure 18. Results of test \# 2. 
SHAPE 1

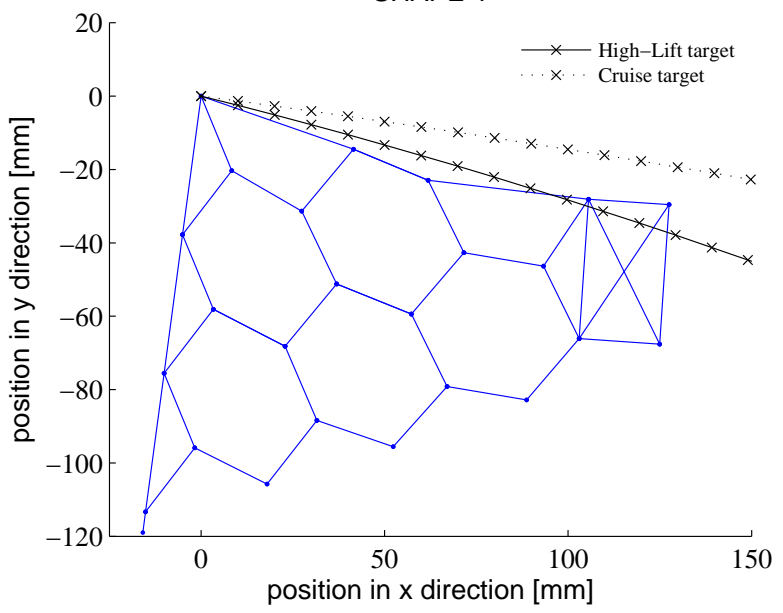

(a) High-lift position
SHAPE 2

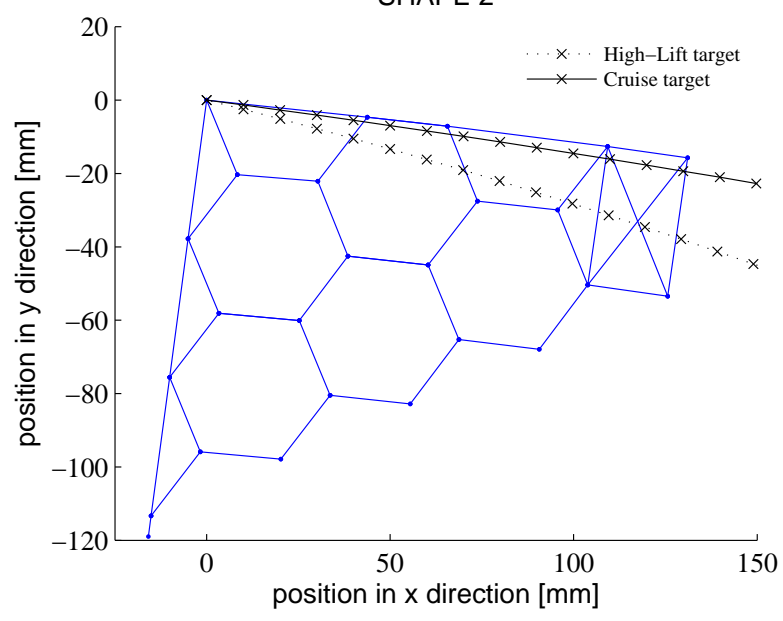

(b) Cruise position

Figure 19. Results of test \# 3 .

The fourth test is a significantly longer PAH section (Figure 20). It can be seen that the calculated shape is not close to target shape 1 in high-lift conditions. The reason for this, is that a longer and thinner PAH section suffers from a numerical instability. How to get to a stable and realistic solution is subject of further study. It is anticipated that this can be done by applying a lower CDP at shape 1 (currently at $7 \mathrm{kPa}$ ) and a higher initial curvature ( $\varphi_{0}$ distribution).

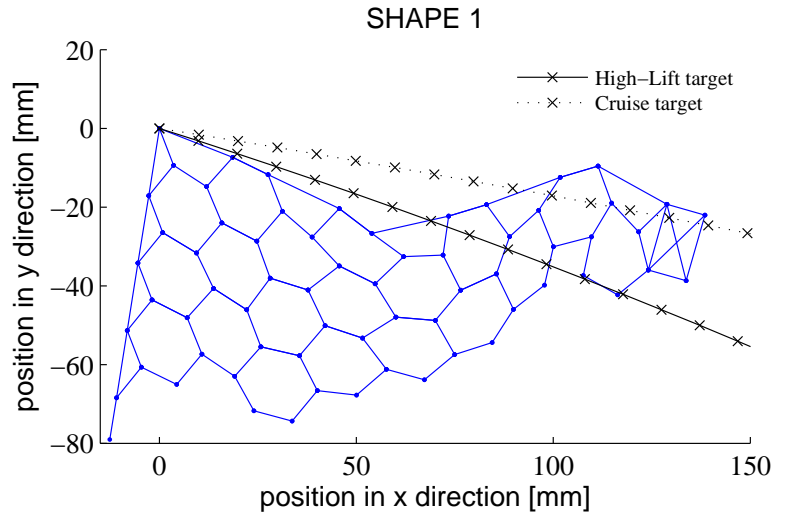

(a) High-lift position

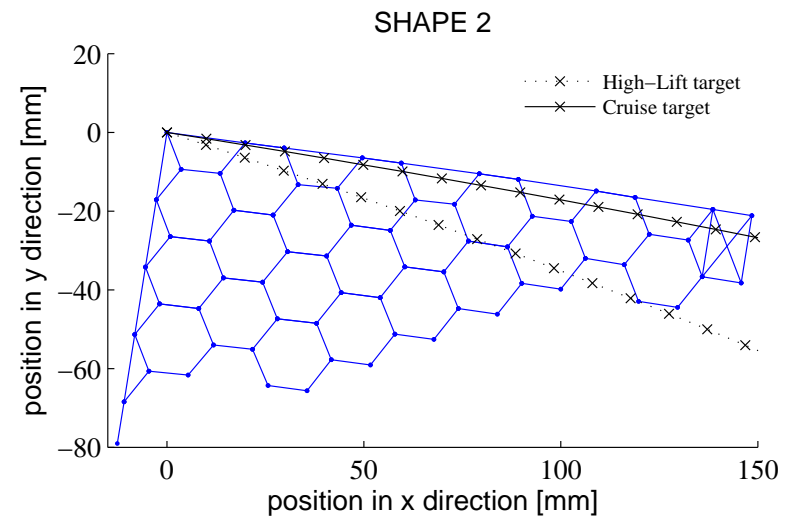

(b) Cruise position

Figure 20. Results of test \# 4.

Based on the results from these four tests, a few observations can be stated. The goal of PAH is to locally change the camber of the flap. Based on test 1 and 2 a relatively short block of honeycomb is sufficient to achieve this. The flap trailing edge does not necessarily need to be completely filled with honeycomb celss. The number of cells is not important for the effectiveness of the flap as long as there are not too few (as in test 3). The deformation should be evenly distributed across the structure in order to get a smooth transfer from one shape to the other and vice versa. This requires a sufficiently fine honeycomb grid relative to the size of the PAH structure.

Finally, the output in terms of skin thickness is presented in Fig. 21. This output is automatically calculated by the tool and it shows how the skin should be manufactured if one is to attain the shapes of Figs. ?? through 20. One can see the discrete steps at the individual nodes. In practice, one might want to limit the individual steps for manufacturing reasons. In the present demonstration one might see the large skin thickness near the root of the PAH section. This is attributed to the large initial angle that is to be 
achieved here (see $\varphi_{0}$ in Table 3 ).

Test 1

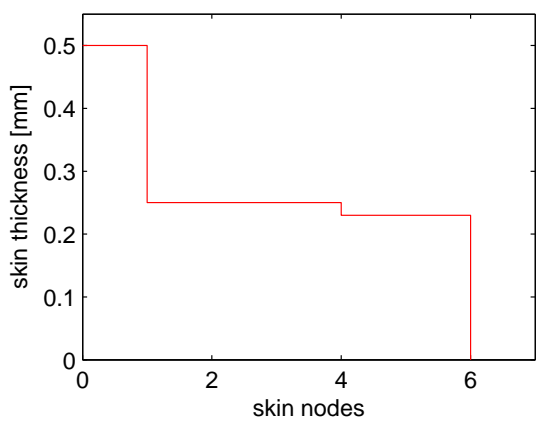

Test 3

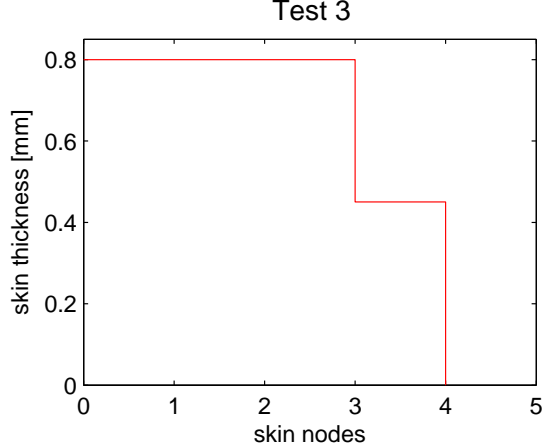

Test 2

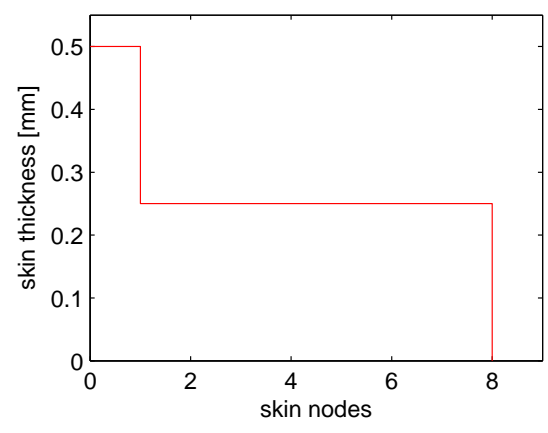

Test 4

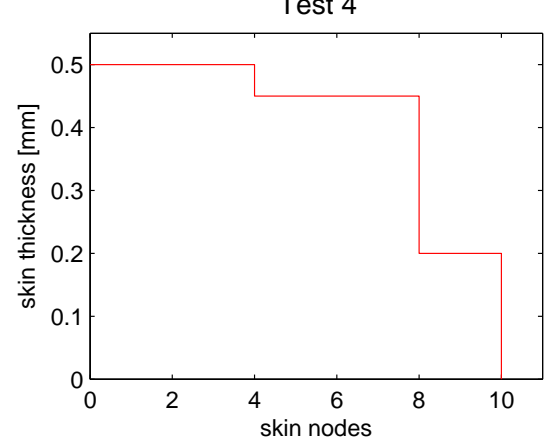

Figure 21. The calculated thickness distribution for the test results.

\section{Limitations and Further Development}

Even though the PAH tool in its current form can be used to aid the designer in designing a pressure-adaptive flap, there is still a lot of effort required to come up with a successful topology. The following limitations are identified:

- One of the limitations of the model is that the same CDP is applied to all cells. Extending the model to accommodate multiple pressure sections might help to solve this problem.

- The manufacturing curvature of the skin is an input to the tool. Eventually this should become a result of the optimization process, together with the desired skin thickness distribution.

- There is currently no aeroelastic loop that calculates the change in pressure distribution on the flap at each calculated shape. Introducing this loop would give a realistic pressure load over the flap.

- No bottom skin is currently present in the present designs. It is anticipated that the bottom skin will be made from a flexible material (such as rubber). The introduction of a flexible skin will introduce more forces in the structure that needs to be accounted for in the simulation.

Each of the items mentioned above is tackled in the proceeding development of the tool.

\section{CONCLUSIONS}

A new design tool for a morphing Fowler flap has been introduced, based on the implementation of pressure-adaptive honeycomb in the aft $30 \%$ of the flap chord. This tool can be used to calcuate the manufacturing topology of the pressure-adaptive honeycomb and skin, such that it can deform between two predefined shapes under aerodynamic loading. It has been shown that the finite-element model that the design tool is based on can successfully predict the mechanics of pressure-adaptive honeycomb. The model showed excellent correlation with the analytical theory and good correlation to experiments. A 
demonstration of the design tool showed how the designer could employ this tool to calculate the desired honeycomb and skin topology that would lead to two distinct predefined target shapes. One in high-lift conditions (with associated aerodynamic pressure distribution) and one in cruise condition (with a different pressure distribution). It showed how the size of the honeycomb cells, the applied pressure differential, and the manufactured skin curvature all contributed to how well the honeycomb was capable of approaching the two target shapes. Further improvements, were also identified to reduce the input of design tool and decrease the design time of an effective pressure-adaptive Fowler flap even further.

\section{ACKNOWLEDGEMENTS}

The authors would like to acknowledge the valuable advice of Dr. Markus Pagitz with respect to the implementation a finite-element model in the design tool. Furthermore, the authors would like to acknowledge the support of the Transport Research Institute of The University of Kansas for providing the authors the ability to investigate pressure adaptive honeycombs and its applications.

\section{References}

${ }^{1}$ Vos, R., Mechanics and Applications of Pressure Adaptive Honeycomb, Ph.D. thesis, University of Kansas, 2009.

${ }^{2}$ Gunston, B., Airbus: The Complete Story, Haynes Publishing, February 2010.

${ }^{3}$ Harris, T. and Lowry, J., "Pressure distribution over an NACA 23012 airfoil with a fixed slot and slotted flap," Technical Memorandum 732, NACA; Langley Memorial Aeronautical Laboratory, Langley Field, Virginia, July 1941.

${ }^{4}$ Johnson, W. and Hill, A., "Pressure distributions from high Reynolds number tests of a Boeing BAC 1 airfoil in the Langley 0.3-meter transonic cryogenic tunnel," Technical Memorandum NASA-TM-87600, NASA; Langley Research Center, Hampton, Virginia, December 1985.

${ }^{5}$ Felippa, C., Nonlinear Finite Element Methods, University of Colorado, Boulder, Colorado, 2001.

${ }^{6}$ Gibson, L. and Ashby, M., Cellular Solids; Structures and Properties, Pergamon Press, Oxford, United Kingdom, 1988.

${ }^{7}$ Warren, W. and Byskov, E., "Three-Fold Symmetry Restrictions on Two-Dimensional Micropolar Materials," European Journal of Mechanics - A/Solids, Vol. 21, No. 5, June 2002, pp. 779-792.

${ }^{8}$ Park, S. and Gao, X.-L., "Micromechanical Modeling of Honeycomb Structures Based on a Modified Couple Stress Theory," Mechanics of Advanced Materials and Structures, Vol. 15, No. 8, November 2008, pp. 574-593.

${ }^{9}$ Gere, J., Mechanics of Materials; 5th edition, Nelson Thornes, Cheltenham, United Kingdom, 2001.

${ }^{10}$ Ferreira, A. and Gladwell, G., MATLAB Codes for Finite Element Analysis: Solids and Structures, Springer, Dordrecht, The Netherlands, 2009. 